\title{
Do measures of surgical effectiveness at 1 year after lumbar spine surgery accurately predict 2 -year outcomes?
}

\author{
Owoicho Adogwa, MD, MPH, ${ }^{1}$ Aladine A. Elsamadicy, BE, ${ }^{1}$ Jing L. Han, BA, ${ }^{1}$ \\ Joseph Cheng, MD, MS, ${ }^{2}$ Isaac Karikari, MD, ${ }^{1}$ and Carlos A. Bagley, MD ${ }^{1}$ \\ 1Division of Neurosurgery, Duke University Medical Center, Durham, North Carolina; and 'Department of Neurosurgery, \\ Vanderbilt University Medical Center, Nashville, Tennessee
}

\begin{abstract}
OBJECTIVE With the recent passage of the Patient Protection and Affordable Care Act, there has been a dramatic shift toward critical analyses of quality and longitudinal assessment of subjective and objective outcomes after lumbar spine surgery. Accordingly, the emergence and routine use of real-world institutional registries have been vital to the longitudinal assessment of quality. However, prospectively obtaining longitudinal outcomes for patients at 24 months after spine surgery remains a challenge. The aim of this study was to assess if 12-month measures of treatment effectiveness accurately predict long-term outcomes (24 months).
\end{abstract}

METHODS A nationwide, multiinstitutional, prospective spine outcomes registry was used for this study. Enrollment criteria included available demographic, surgical, and clinical outcomes data. All patients had prospectively collected outcomes measures and a minimum 2-year follow-up. Patient-reported outcomes instruments (Oswestry Disability Index [ODI], SF-36, and visual analog scale [VAS]-back pain/leg pain) were completed before surgery and then at 3, 6, 12, and 24 months after surgery. The Health Transition Index of the SF-36 was used to determine the 1- and 2-year minimum clinically important difference (MCID), and logistic regression modeling was performed to determine if achieving MCID at 1 year adequately predicted improvement and achievement of MCID at 24 months.

RESULTS The study group included 969 patients: 300 patients underwent anterior lumbar interbody fusion (ALIF), 606 patients underwent transforaminal lumbar interbody fusion (TLIF), and 63 patients underwent lateral interbody fusion (LLIF). There was a significant correlation between the 12- and 24-month ODI ( $r=0.82 ; p<0.0001)$, SF-36 Physical Component Summary score ( $r=0.89 ; p<0.0001)$, VAS-back pain $(r=0.90 ; p<0.0001)$, and VAS-leg pain $(r=0.85 ; p<$ $0.0001)$. For the ALIF cohort, patients achieving MCID thresholds for ODI at 12 months were 13 -fold $(p<0.0001)$ more likely to achieve MCID at 24 months. Similarly, for the TLIF and LLIF cohorts, patients achieving MCID thresholds for ODI at 12 months were 13 -fold and 14-fold ( $p<0.0001)$ more likely to achieve MCID at 24 months. Outcome measures obtained at 12 months postoperatively are highly predictive of 24-month outcomes, independent of the surgical procedure.

CONCLUSIONS In a multiinstitutional prospective study, patient-centered measures of surgical effectiveness obtained at 12 months adequately predict long-term (24-month) outcomes after lumbar spine surgery. Patients achieving MCID at 1 year were more likely to report meaningful and durable improvement at 24 months, suggesting that the 12-month time point is sufficient to identify effective versus ineffective patient care.

http://thejns.org/doi/abs/10.3171/2015.8.SPINE15476

KEY WORDS lumbar spine; spinal surgery; surgical effectiveness; surgical outcomes; effective measures; long-term outcomes

$\mathrm{L}$ UMBAR spinal stenosis is a recognized cause of lowback and radicular pain, with far reaching effects on our health care system. ${ }^{6,8,13}$ The socioeconomic impact is staggering, and direct medical costs have tripled in the past decade. ${ }^{14,16,18}$ Concurrently, the rates of lumbar fusions for degenerative lumbar disease have quadrupled, contributing to a significant increase in back-related health care utilization and costs..$^{14,18}$ In an effort to control spiraling health care costs, improve treatment effectiveness, and maximize patient quality of life, prospective registries have emerged as a feasible way to capture real-world spine care across large patient populations.

ABBREVIATIONS ALIF = anterior lumbar interbody fusion; HRQOL = health-related quality of life; LLIF = lateral interbody fusion; MCID = minimum clinically important difference; $\mathrm{MCS}=$ Mental Component Summary; MDC = minimum detectable change; ODI = Oswestry Disability Index; PCS = Physical Component Summary; TLIF = transforaminal lumbar interbody fusion; VAS = visual analog scale. SUBMITTED April 24, 2015. ACCEPTED August 24, 2014.

INCLUDE WHEN CITING Published online January 1, 2016; DOI: 10.3171/2015.8.SPINE15476. 
Prospective registries measure treatment effectiveness and quality for real-world patients in real-world clinical settings. ${ }^{12}$ National and international registries facilitate reliable and efficient access to patients' clinical and functional outcomes data, such as quality of life and overall patient satisfaction with health care received. ${ }^{12}$ Integral to current prospective registry data collection systems are validated patient-reported outcomes measures that assess the effectiveness of care, such as the Oswestry Disability Index (ODI), ${ }^{5} \mathrm{SF}-36,{ }^{21}$ and numeric rating scales for back and leg pain.

Historically, the 2-year patient-reported outcomes have been used as the measure of treatment effectiveness and durability:, ${ }^{1,5,5,12,14}$ however, the insistence of 2-year patient-reported outcomes may be abating as health-related quality-of-life (HRQOL) status at 1 year is increasingly being reported for some lumbar fusion procedures. In general, whether 1-year outcomes adequately predict 2-year outcomes for elective lumbar fusion surgery remains unknown. The purpose of this study was to determine the degree to which 1-year patient-reported outcomes accurately predict 2-year outcomes after lumbar fusion surgery for degenerative lumbar disease.

\section{Methods}

The primary aim of this multicenter study was to assess if 12-month measures of treatment effectiveness accurately predict long-term outcomes (24 months). This study was based on prospectively collected demographic, surgical, and functional outcomes data. The database was queried for all patients undergoing lumbar interbody fusion for symptomatic back and leg pain secondary to degenerative disc disease or Grade I spondylolisthesis who had complete preoperative and 1- and 2-year postoperative patient-reported outcomes measures. Fourteen surgeons from 10 institutions participated in the study. Only patients with comprehensive 2-year data were included in the final analysis.

Institutional review board approval was obtained prior to initiation of the study. We included patients who were 18 to 70 years old with 1) low-back pain with associated radiculopathy; 2) MRI evidence of degenerative disc disease or Grade I spondylolisthesis with central or foraminal stenosis; 3) patients in whom at least 6 weeks of nonsurgical treatment had failed; 4) patients who underwent lumbar spinal fusion (e.g., transforaminal lumbar interbody fusion [TLIF], anterior lumbar interbody fusion [ALIF], or lateral interbody fusion [LLIF]), and 5) patients with available patient-reported outcomes data at baseline and at the 1- and 2-year time points following surgery. Patients were excluded if they had a history of 1) prior back surgery; 2) extraspinal causes of back pain or sciatica; 3 ) severe coexisting pathology that could confound the assessment of operative outcome (e.g., rheumatoid arthritis, osteoarthritis, metabolic bone disease); or 4) were unwilling or unable to participate in follow-up procedures.

\section{Patient-Reported Outcome Measures}

Patient demographics, clinical presentation, indications for surgery, radiological studies, and operative variables were assessed for all patients enrolled in this study. All questionnaires were self-administered and were identical at baseline and 1-, and 2-year follow-ups. Patient-reported outcomes instruments included the ODI low-back disability questionnaire, $, 7,75 \mathrm{SF}-36,{ }^{21}$ and numeric rating scales for back and leg pain. $., 5,17$

The ODI is a self-administered questionnaire that assesses back-specific function and contains 10 questions with 6 response categories. Higher scores denote increasing disability or higher pain levels. Each variable is rated on a 0 - to 5-point scale and transformed into a percentage score. The range of possible values is from 0 to $100(0-20$ = minimal disability; $21-40=$ moderate disability; $41-60$ = severe disability; $61-80=$ crippled; $81-100=$ bedbound ).

The SF-36 is a self-administered short-form health status survey that evaluates physical function, social function, mental health, vitality, bodily pain, and general health. Two composite scores can be calculated: the Physical Component Summary (PCS) and the Mental Component Summary (MCS). Using US normalized-based scoring, all domain scales have a mean of 50 and a standard deviation of 10 . Scores lower than 50 fall below the mean of the general population.

Numeric rating scales for back and leg pain range from 0 to 10 , with 10 being the worst pain imaginable. The aforementioned instruments have been widely used in the spine literature and have shown good construct validity.

At 1 and 2 years postoperatively, patients scored their clinical status on the following scale: 1) "surgery met my expectations;" 2) "I did not improve as much as I had hoped, but I would undergo the same surgery for the same outcome;" 3) "surgery helped, but I would not undergo the same treatment for the same outcome;" or 4) "I am the same as or worse than I was before the surgery." Since patients graded the evolution in their own health status, this classification was considered the external criterion for any change to be "clinically relevant."

\section{Statistical Analysis}

Parametric data were expressed as the mean \pm SD and were compared using the Student t-test. Nonparametric data were expressed as the median (interquartile range) and were compared using the Mann-Whitney U-test. Nominal data were compared using the chi-square test. All tests were 2-sided and were statistically significant if the $\mathrm{p}$ value was less than 0.05 .

Change in health status was calculated at 1 and 2 years postoperatively as the postoperative score minus the preoperative score for each patient-reported outcome measure. Preoperative versus 1-year postoperative and preoperative versus 2-year postoperative health status measures were evaluated using the matched-pairs sample t-test. The mean change in health status measures from 1 to 2 years postoperatively was also assessed. Linear regression modeling was used to determine the correlation between changes in the 1- and 2-year health status scores.

The threshold values for the minimum clinically important difference (MCID) were calculated for each patient cohort (ALIF, TLIF, and LLIF). MCID is the smallest change in an outcome measure that represents a change that would be considered meaningful by the patient. MCID values were calculated for all health status measures. Dif- 
ferent approaches can be used to determine MCID. One approach is to use receiver operating characteristic curves to define the cutoff point that best discriminates between patients reporting or denying any improvement. Another is to estimate the mean change score in patients who actually report improvement from baseline. The method used to determine the MCID in the most often cited publication was the minimum detectable change method (MDC). The MDC is an extension of the least significant difference concept and is the smallest change that is above measurement error within a confidence interval. The MDC was calculated as $1.96 \times \sqrt{ } 2 \times$ SEM.$^{20}$ The SEM was estimated by taking the square root of the within-subject variance (which consists of variance between measures plus the residual variance on a 2-way ANOVA random effects model) of patients categorized as "unchanged" by the external criteria. MDC can be interpreted as the magnitude of the change below which there is more than a $95 \%$ chance that no real change has occurred. All analyses were performed using SAS 9.3 (SAS Institute, Inc.).

\section{Results \\ Patient Population}

Of the initially enrolled 2000 patients, a total of 969 patients had comprehensive 2-year follow-up data. Overall, the mean age at the time of surgery was $52.35 \pm 12.19$ years (ALIF $47.92 \pm 11.33$ years; TLIF $52.14 \pm 12.50$ years; LLIF $56.48 \pm 12.51$ years). There were 375 (38.75\%) men and $594(61.25 \%)$ women. The median number of levels fused was 2 (interquartile range 1-3). All patients presented with symptomatic back and leg pain secondary to degenerative disc disease or Grade I spondylolisthesis. Overall, the mean preoperative visual analog scale (VAS)-back pain, VAS-leg pain, and ODI scores were $7.00 \pm 2.50,6.79$ \pm 3.01 , and $47.28 \pm 15.52$, respectively. The mean preoperative SF-36 MCS and SF-36 PCS scores were $39.74 \pm$ 17.28 and $27.12 \pm 10.52$, respectively. The demographic and baseline characteristics are presented in Table 1 .

\section{Postoperative Outcomes}

Complete baseline and 2-year outcomes data were available for all patients enrolled in this study. There was a significant improvement from baseline in all patient-re-
TABLE 2. Changes in baseline characteristics of the patients undergoing index ALIF, TLIF, or LLIF surgery*

\begin{tabular}{cccc}
\hline $\begin{array}{c}\text { Change From } \\
\text { Baseline in Patient- } \\
\begin{array}{c}\text { Reported Outcomes } \\
\text { Measures }\end{array}\end{array}$ & ALIF $(n=300)$ & TLIF $(n=606)$ & LLIF $(n=63)$ \\
\hline At 1 yr & & & \\
\hline VAS-BP & $3.78 \pm 2.85$ & $3.81 \pm 2.78$ & $3.79 \pm 2.94$ \\
\hline VAS-LP & $3.51 \pm 3.15$ & $3.18 \pm 2.97$ & $3.54 \pm 3.03$ \\
\hline SF-36 MCS & $42.14 \pm 17.15$ & $44.29 \pm 16.17$ & $44.45 \pm 19.19$ \\
\hline SF-36 PCS & $35.01 \pm 14.18$ & $35.97 \pm 13.44$ & $31.71 \pm 15.09$ \\
\hline ODI & $30.38 \pm 21.08$ & $30.43 \pm 19.88$ & $30.36 \pm 19.24$ \\
\hline At 2 yrs & & & \\
\hline VAS-BP & $4.29 \pm 3.25$ & $4.29 \pm 2.89$ & $4.00 \pm 2.97$ \\
\hline VAS-LP & $3.94 \pm 3.25$ & $3.67 \pm 3.09$ & $3.07 \pm 3.25$ \\
\hline SF-36 MCS & $42.63 \pm 16.34$ & $42.64 \pm 18.03$ & $45.46 \pm 17.95$ \\
\hline SF-36 PCS & $34.84 \pm 13.79$ & $33.62 \pm 14.96$ & $32.09 \pm 14.05$ \\
\hline ODI & $32.70 \pm 21.46$ & $32.53 \pm 21.14$ & $31.98 \pm 19.34$ \\
\hline Al & & & \\
\hline
\end{tabular}

* All cohorts were matched at baseline. Values given as the mean \pm standard deviation.

ported outcome metrics at 1 and 2 years postoperatively (Table 2, Fig. 1). Overall, the mean 1-year improvements from baseline in VAS-BP, VAS-LP, and ODI scores were $3.35 \pm 3.70,3.41 \pm 3.99$, and $20.35 \pm 24.08(\mathrm{p}=0.001)$, respectively (Table 2, Fig. 1). The mean 2-year improvements from baseline in VAS-BP, VAS-LP, and ODI scores were $2.34 \pm 3.56,2.51 \pm 3.99$, and $13.09 \pm 21.91(p=0.012)$, respectively (Table 2, Fig. 1). The mean SF-36 PCS score was $27.12 \pm 10.52$ preoperatively and improved to $33.20 \pm$ 9.15 at 1 year and to $33.51 \pm 9.15$ at 2 years (Table 2, Fig. 1). Paired-sample analysis was performed by comparing the mean 1- and 2-year patient-reported outcome scores. There was no statistically significant difference between the 1-year and 2-year scores for VAS-BP $(p=0.47)$, VASLP $(p=0.23)$, ODI $(p=0.56)$, and SF-36 PCS $(p=0.85)$. Similarly, a paired-sample t-test for each subgroup (ALIF, TLIF, and LLIF) comparing 1-year versus 2-year postoperative scores revealed no statistically significant difference for any of the HRQOL measures.

TABLE 1. Baseline characteristics and patients undergoing index ALIF, TLIF, or LLIF surgery*

\begin{tabular}{|c|c|c|c|c|c|}
\hline Variable & Combined Cohort $(n=969)$ & ALIF $(n=300)$ & TLIF $(n=606)$ & LLIF $(n=63)$ & $\mathrm{p}$ Value \\
\hline Patient age in yrs & $52.35 \pm 12.19$ & $47.92 \pm 11.33$ & $52.14 \pm 12.50$ & $56.48 \pm 12.51$ & 0.457 \\
\hline Body mass index in $\mathrm{kg} / \mathrm{m}^{2}$ & $29.74 \pm 6.19$ & $29.23 \pm 5.85$ & $30.16 \pm 6.44$ & $29.62 \pm 5.56$ & 0.180 \\
\hline Male $(\%)$ & 38.75 & 30.13 & 43.07 & 43.07 & 0.035 \\
\hline \multicolumn{6}{|c|}{ Preop patient-reported outcomes } \\
\hline VAS-BP & $7.00 \pm 2.50$ & $7.16 \pm 2.41$ & $6.87 \pm 2.56$ & $7.07 \pm 2.14$ & 0.134 \\
\hline VAS-LP & $6.79 \pm 3.01$ & $6.78 \pm 2.82$ & $6.40 \pm 3.14$ & $7.20 \pm 2.28$ & 0.021 \\
\hline SF-36 MCS & $39.74 \pm 17.28$ & $39.52 \pm 16.20$ & $39.75 \pm 18.07$ & $39.97 \pm 13.42$ & 0.195 \\
\hline SF-36 PCS & $27.12 \pm 10.52$ & $26.87 \pm 10.04$ & $25.33 \pm 10.84$ & $29.16 \pm 7.79$ & 0.102 \\
\hline ODI & $47.28 \pm 15.52$ & $47.86 \pm 14.73$ & $47.15 \pm 16.11$ & $46.85 \pm 14.50$ & 0.613 \\
\hline
\end{tabular}

$\mathrm{BP}=$ back pain; $\mathrm{LP}=$ leg pain.

${ }^{*}$ All cohorts were matched at baseline. Values are given as the mean \pm standard deviation unless indicated otherwise. 

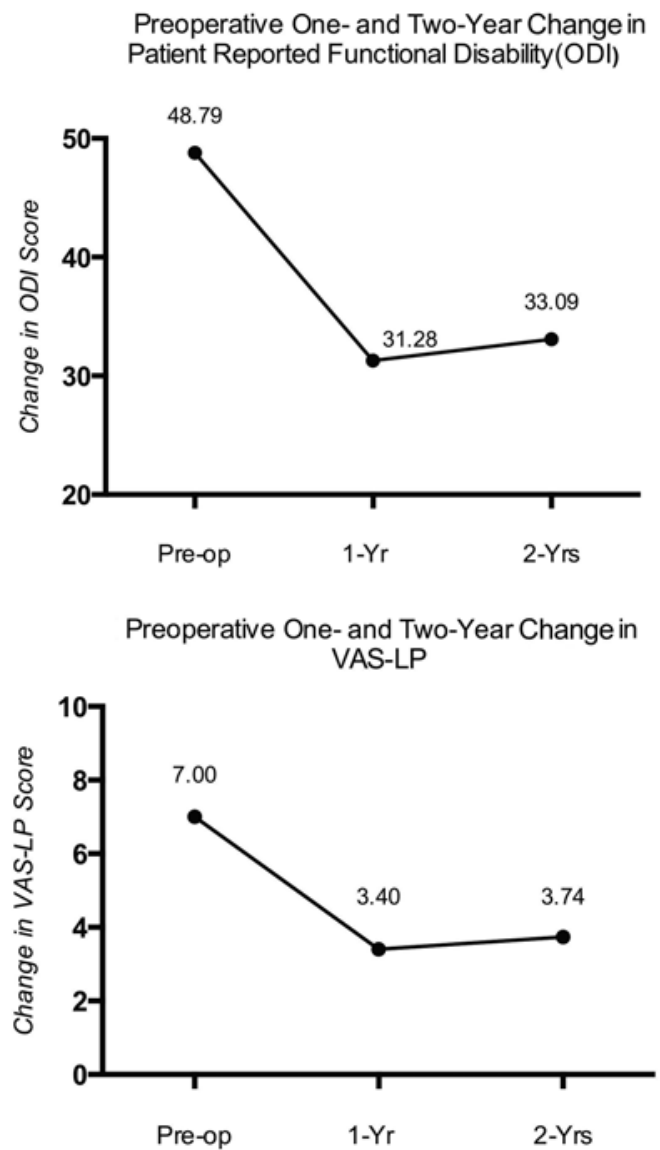

Preoperative One- and Two-Year Change in
VAS-BP

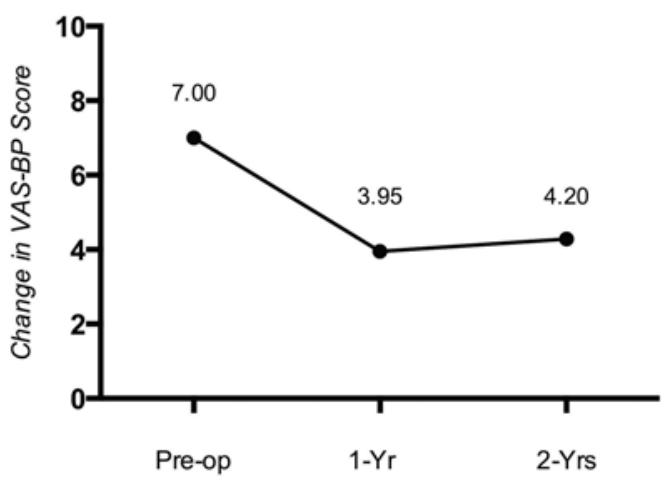

Preoperative One- and Two-Year Change in SF-36 PCS

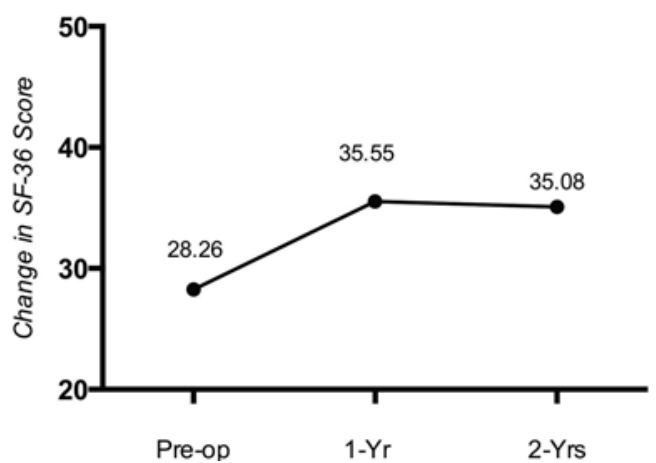

FIG. 1. One- and 2-year changes from baseline in patient-reported outcomes measures. In all HRQOL measures, patients experienced a statistically significant improvement from baseline with the greatest improvement occurring within the 1st postoperative year. $\mathrm{BP}=$ back pain; $\mathrm{LP}=$ leg pain.

\section{Meaningful Improvement}

The threshold values for MCID were calculated for each patient cohort (ALIF, TLIF, and LLIF) at 1 and 2 years postoperatively. The MDC-derived MCID thresholds for the ALIF cohort at 1 year postoperatively was 3.1 for VAS-BP, 3.0 for VAS-LP, 9.0 for ODI, and 12 for SF-36 PCS. At 2 years, the MCID thresholds were 2.9 for VAS-BP, 2.7 for VAS-LP, 11 for ODI, and 8.0 for SF-36 PCS. Seventy percent of patients at 1 year reported a level of improvement after surgery that met their expectations (meaningful improvement) and 65\% of patients achieved MCID thresholds at 2 years (treatment durability) (Fig. 2).

The MDC-derived MCID thresholds for the TLIF cohort at 1 year postoperatively was 3.0 for VAS-BP, 2.9 for VAS-LP, 7.7 for ODI, and 9.0 for SF-36 PCS. At 2 years, the MCID thresholds were 3.2 for VAS-BP, 2.8 for VASLP, 9.0 for ODI, and 10 for SF-36 PCS. Seventy-nine percent of patients at 1 year reported a level of improvement after surgery that met their expectations (meaningful improvement), and $62 \%$ of patients achieved MCID thresholds at 2 years (treatment durability) (Fig. 3).

The MDC-derived MCID thresholds for the LLIF cohort at 1 year postoperatively were 2.7 for VAS-BP, 3.0 for VAS-LP, 8.0 for ODI, and 10.0 for SF-36 PCS. At 2 years, the MCID thresholds were 2.6 for VAS-BP, 2.8 for VASLP, 10 for ODI, and 7.7 for SF-36 PCS. Seventy-seven per- cent of patients at 1 year reported a level of improvement after surgery that met their expectations (meaningful improvement), and $75 \%$ of patients achieved MCID thresholds at 2 years (treatment durability) (Fig. 4).

The aforementioned MCID values are within the reference ranges previously reported in the literature..$^{5,10,19}$

\section{Predictive Value of 1-Year Patient-Reported Outcomes}

Logistic regression analysis was used to assess if the 12-month measures of treatment effectiveness accurately predict long-term outcomes (24 months). Overall, patients achieving MCID thresholds at 1 year were more likely to achieve MCID thresholds at 2 years. In the subgroup analysis, patients in the ALIF cohort who achieved MCID thresholds at 1 year were 12-fold more likely to achieve MCID thresholds at 2 years for ODI, 9-fold more likely to achieve MCID thresholds at 2-years for SF-36 PCS, and 6-fold more likely to achieve MCID thresholds at 2 years for VAS-BP and VAS-LP. Patients in the TLIF cohort who achieved MCID thresholds at 1 year were 13fold more likely to achieve MCID thresholds at 2 years for ODI, 8-fold more likely to achieve MCID thresholds at 2 years for SF-36 PCS, 7-fold more likely to achieve MCID thresholds at 2 years for VAS-BP, and 6-fold more likely to achieve MCID thresholds for VAS-LP. Similarly, among patients in the LLIF cohort, patients who achieved MCID 


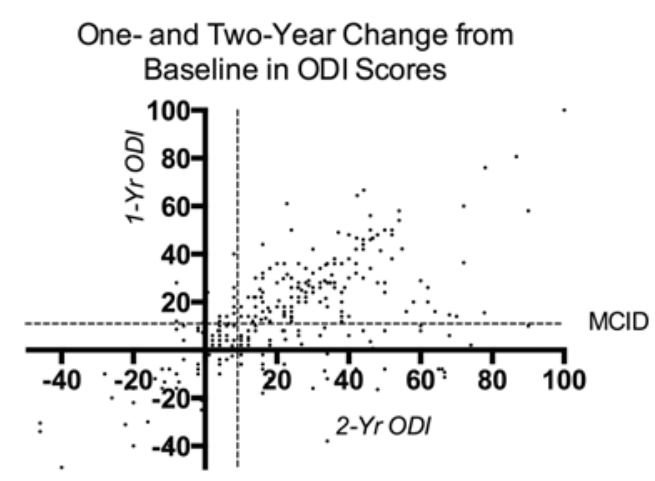

One- and Two-Year Change from

Baseline in VAS LP Scores

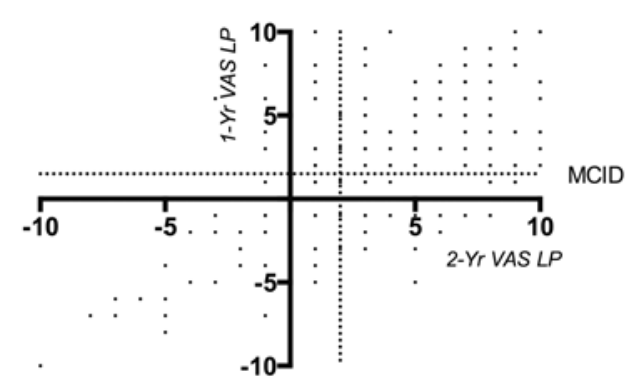

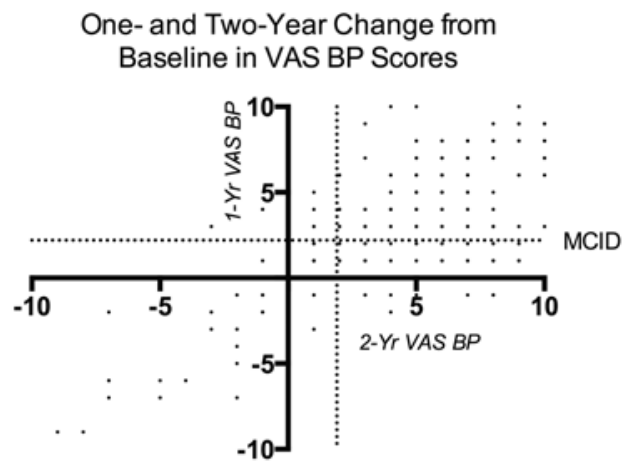

One- and Two-Year Change from Baseline in SF-36 PCS

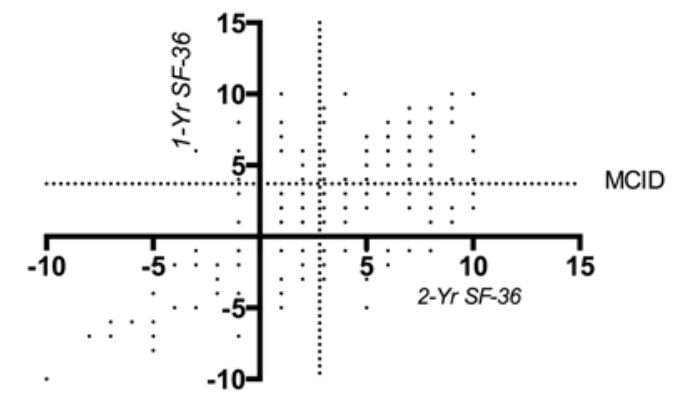

FIG. 2. The MDC-derived MCID thresholds for the ALIF cohort at 1 and 2 years postoperatively. Seventy percent of patients at 1 year reported a level of improvement after surgery that met their expectations (meaningful improvement), and $65 \%$ of patients achieved MCID thresholds at 2 years.

thresholds at 1 year were 14 -fold more likely to achieve MCID thresholds at 2 years for ODI, 8 -fold more likely to achieve MCID thresholds at 2-years for SF-36 PCS, 5-fold more likely to achieve MCID thresholds at 2 years for VAS-BP, and 4-fold more likely to achieve MCID thresholds for VAS-LP (Table 3).

\section{Discussion}

Changes in HRQOL measures, particularly diseasespecific SF-36 and ODI, have become important standards for the evaluation of lumbar spine surgery outcomes. In this multiinstitutional prospective study, patient-centered measures of surgical effectiveness obtained at 12 months adequately predict long-term (24 months) outcomes after lumbar spine surgery. We demonstrate that patients achieving MCID thresholds at 1 year were more likely to report meaningful and durable improvement at 2 years, suggesting that the 12-month measures of treatment effectiveness are sufficient to identify effective versus ineffective patient care.

As has been well demonstrated, lumbar interbody fusions for degenerative spinal disorders have been shown to be clinically and cost-effective treatments. ${ }^{2,4,6,9} \mathrm{~A}$ recent study by Glassman et al. demonstrated that lumbar fusion is a highly effective, durable, and cost-effective treatment at 5 years after index surgery. ${ }^{11}$ Similarly, Adogwa et al. demonstrated in a 2-year prospective study that lumbar fusions are clinically efficacious and highly cost-effective., ${ }^{1,2}$ In the current study, the majority of patients $(>75 \%)$ undergoing index lumbar fusions reported a statistically sig- nificant improvement from baseline in all patient-reported outcomes measures, except SF-36 MCS. Mental health status appears to be more refractory to surgery. Furthermore, the statistically significant improvement observed in all patient-assessed outcomes metrics was independent of surgical procedure (ALIF, TLIF, LLIF).

Not surprisingly, most of the observed changes from baseline in HRQOL metrics occurred over the 1st postoperative year after index lumbar spine surgery; ${ }^{10,11}$ accordingly, across patient cohorts, less change in HRQOL outcomes was observed between 1 and 2 years postoperatively. In fact, in some metrics, we observed mild deterioration in patient-centered measures of surgical effectiveness between the 1- and 2-year postoperative intervals. Prior studies have reported similar results. In a recent study by Glassman et al., 12-month HRQOL measures were compared with 24-month HRQOL measures in 283 patients undergoing adult deformity surgery. ${ }^{11}$ The authors reported statistically significant improvements in patient-reported outcomes metrics at the 12-month postoperative interval; however, for most patients in the study, the outcome scores stabilized after the 1-year postoperative interval. The stabilization in patient-reported outcomes suggest that 1 year after surgery appears to be a reasonable time frame to anticipate maximal improvement after index lumbar spine surgery.

Measures of treatment effectiveness do not always correlate to clinically meaningful results. Because of this, the concept of MCID was developed as a means to measure the critical threshold of improvement needed to achieve 


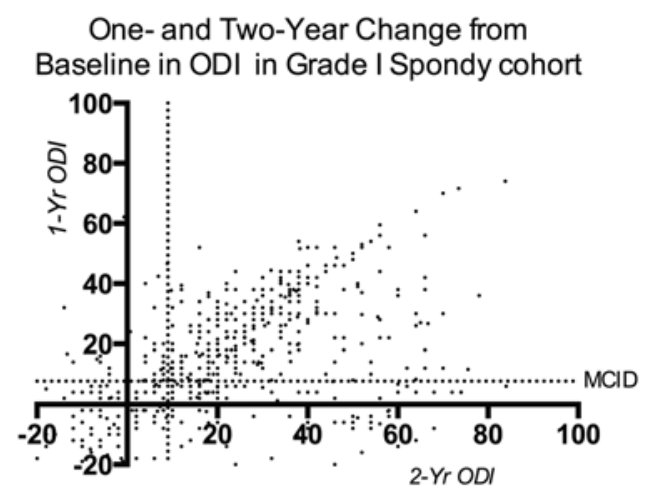

One- and Two-Year Change from Baseline in VAS LP

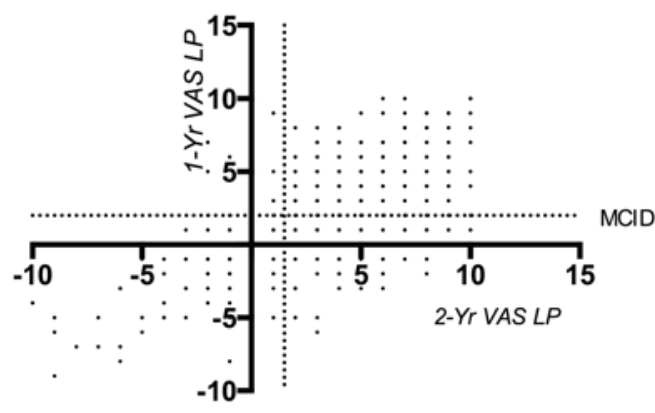

One- and Two-Year Change from Baseline in VAS BP

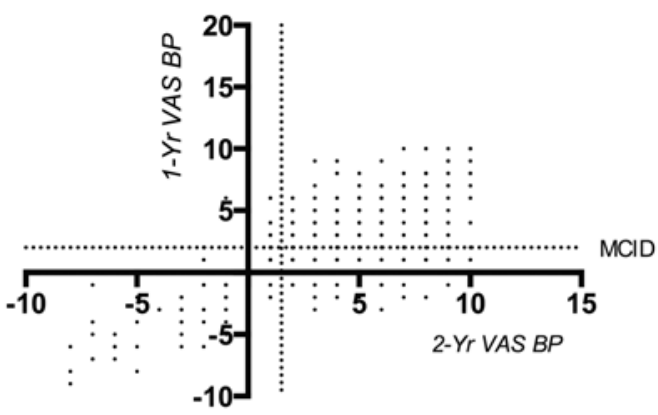

One- and Two-Year Change from Baseline in SF-36

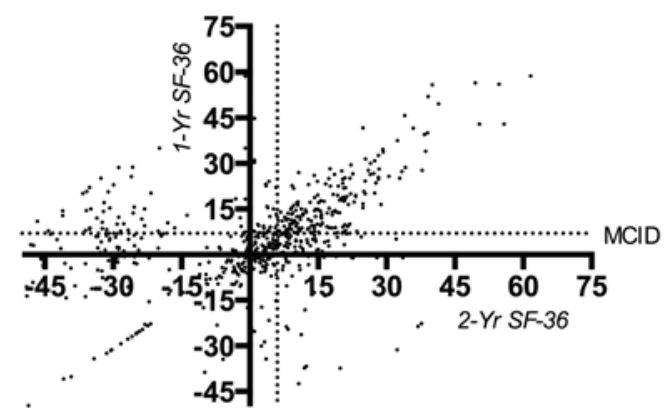

FIG. 3. The MDC-derived MCID thresholds for the TLIF cohort at 1 and 2 years postoperatively. Seventy-nine percent of patients at 1 year reported a level of improvement after surgery that met their expectations (meaningful improvement), and $62 \%$ of patients achieved MCID threshold at 2 years. Spondy = spondylolisthesis.

clinically meaningful treatment effectiveness. ${ }^{5,10}$ Accordingly, MCID is a quantitative assessment of clinical relevance for a given magnitude of change in the HRQOL scores. Different approaches can be used to determine MCID; however, no consensus has been reached as to the superior method with which to calculate MCID. For this study, the MDC method was used..$^{5}$ The choice of MDC has the advantage of eliminating potential MCID thresholds that are within the measurement error. Furthermore, in several recent studies, the MDC approach has been shown to be the superior calculation method for determining MCID thresholds in patients undergoing various lumbar spine surgeries.

The MCID thresholds at 2 years following ALIF were 2.9 for VAS-back pain, 2.7 for VAS-leg pain, 11 for ODI, and 8.0 for SF-36 PCS, with $70 \%$ of patients achieving MCID thresholds at 1 year and $65 \%$ of patients achieving MCID thresholds at 2 years postoperatively. The MCID thresholds at 2 years following TLIF were 3.2 for VASback pain, 2.8 for VAS-leg pain, 9.0 for ODI, and 10 for SF36 PCS, with 79\% of patients achieving MCID thresholds at 1 year and $62 \%$ of patients achieving MCID thresholds at 2 -years postoperatively. Similarly, the MCID thresholds at 2 years following LLIF were 2.6 for VAS-back pain, 2.8 for VAS-leg pain, 10 for ODI, and 7.7 for SF-36 PCS, with 77\% of patients achieving MCID thresholds at 1 year and $75 \%$ of patients achieving MCID thresholds at 2 years postoperatively. MCID thresholds for treatment effectiveness have been previously reported as being improvements in VAS-
TABLE 3. Logistic regression analysis of 1-year patient-reported outcomes scores predicting 2-year improvement in pain and functional disability

\begin{tabular}{cccc}
\hline Variable & OR & $95 \% \mathrm{Cl}$ & p Value \\
\hline ALIF cohort & & & \\
\hline ODI & 12.481 & $10.587-14.714$ & $<0.0001$ \\
\hline SF-36 PCS & 9.166 & $7.812-10.756$ & $<0.0001$ \\
\hline VAS-BP & 6.315 & $5.450-7.316$ & $<0.0001$ \\
\hline VAS-LP & 5.781 & $4.990-6.698$ & $<0.0001$ \\
\hline TLIF cohort† & & & \\
\hline ODI & 12.978 & $10.981-15.337$ & $<0.0001$ \\
\hline SF-36 PCS & 8.776 & $7.453-10.334$ & $<0.0001$ \\
\hline VAS-BP & 6.850 & $5.893-7.962$ & $<0.0001$ \\
\hline VAS-LP & 6.150 & $5.276-7.168$ & $<0.0001$ \\
\hline LLIF cohort & & & \\
\hline ODI & 13.505 & $11.420-15.970$ & $<0.0001$ \\
\hline SF-36 PCS & 8.211 & $7.022-9.601$ & $<0.0001$ \\
\hline VAS-BP & 5.450 & $5.450-7.316$ & $<0.0001$ \\
\hline VAS-LP & 4.724 & $4.094-5.450$ & $<0.0001$ \\
\hline
\end{tabular}

* At 1 year, patients in the ALIF cohort reporting changes in the ODI scores exceeding the calculated MCID scores were 12 -fold more likely to achieve $M C I D$ at 2 years after index lumbar surgery.

$\dagger$ Patients in the TLIF and LLIF cohorts who achieved 1-year MCID were 13and 14 -fold more likely to achieve MCID at 2 years, respectively. 

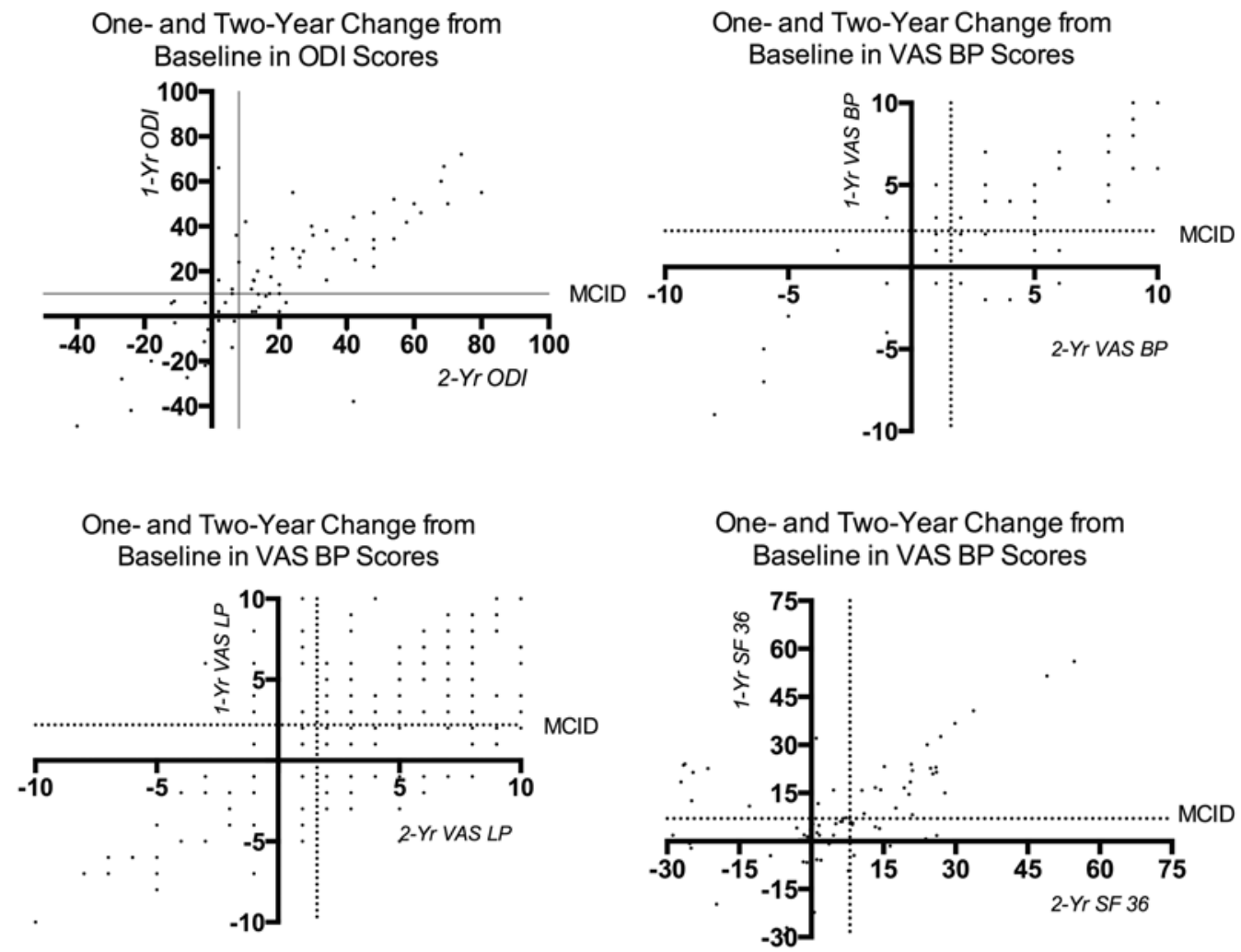

FIG. 4. The MDC-derived MCID thresholds for the LLIF cohort at 1 and 2 years postoperatively. Seventy-seven percent of patients at 1 year reported a level of improvement after surgery that met their expectations (meaningful improvement), and $75 \%$ of patients achieved MCID thresholds at 2 years.

back pain of 1 to 4 points, VAS-leg pain of 1 to 3 points, ODI of 6 to 19 points, and SF-36 PCS of 7 to 22 points., ${ }^{5,10,11}$

Overall, patients achieving MCID thresholds at 1 year were more likely to achieve MCID thresholds at 2 years. Across patient cohorts, the patients' MCID thresholds at 1 year were 12 - to 14 -fold more likely to achieve MCID thresholds for ODI at 2 years, 8- to 9-fold more likely to achieve MCID thresholds for SF-36 PCS at 2 years, and 4- to 7-fold more likely to achieve MCID thresholds for VAS-back/leg pain at 2 years. This implies that the majority of patients reporting a significant clinical benefit at 1 year continue to report treatment effectiveness at 2 years.

There are limitations to the present study. Intraoperative and postoperative therapy was left up to the clinical judgment of the operating surgeon; different rehabilitation strategies could potentially lead to different outcomes. The effect of perioperative complications was not included in the analysis. Furthermore, the occurrence of a complication could affect an individual's ability to achieve MCID. Finally, it remains unknown whether patients who had inferior outcomes were followed by other providers not associated with this study. Despite these limitations, the current study demonstrates that the 12-month measure of treatment effectiveness is sufficient to identify effective versus ineffective patient care.

\section{Conclusions}

In a multiinstitutional prospective study, patient-centered measures of surgical effectiveness obtained at 12 months adequately predict long-term (24-month) outcomes after lumbar spine surgery. Patients achieving MCID at 1 year were more likely to report meaningful and durable improvement by 24 months, suggesting that the 12-month time-point is sufficient to identify effective versus ineffective patient care.

\section{References}

1. Adogwa O, Parker SL, Bydon A, Cheng J, McGirt MJ: Comparative effectiveness of minimally invasive versus open transforaminal lumbar interbody fusion: 2-year assessment of narcotic use, return to work, disability, and quality of life. $\mathbf{J}$ Spinal Disord Tech 24:479-484, 2011

2. Adogwa O, Parker SL, Davis BJ, Aaronson O, Devin C, Cheng JS, et al: Cost-effectiveness of transforaminal lumbar interbody fusion for Grade I degenerative spondylolisthesis. J Neurosurg Spine 15:138-143, 2011

3. Adogwa O, Parker SL, Mendenhall SK, Shau DN, Aaronson $\mathrm{O}$, Cheng J, et al: Laminectomy and extension of instrumented fusion improves 2-year pain, disability, and quality of life in patients with adjacent segment disease: defining the long-term effectiveness of surgery. World Neurosurg 80:893-896, 2013

4. Christensen A, Høy K, Bünger C, Helmig P, Hansen ES, Andersen T, et al: Transforaminal lumbar interbody fusion vs. posterolateral instrumented fusion: cost-utility evaluation along side an RCT with a 2-year follow-up. Eur Spine J 23:1137-1143, 2014

5. Copay AG, Glassman SD, Subach BR, Berven S, Schuler TC, Carreon LY: Minimum clinically important difference in lumbar spine surgery patients: a choice of methods using the 
Oswestry Disability Index, Medical Outcomes Study questionnaire Short Form 36, and pain scales. Spine J 8:968-974, 2008

6. Crandall DG, Revella J: Transforaminal lumbar interbody fusion versus anterior lumbar interbody fusion as an adjunct to posterior instrumented correction of degenerative lumbar scoliosis: three year clinical and radiographic outcomes. Spine (Phila Pa 1976) 34:2126-2133, 2009

7. Dailey AT, Ghogawala Z, Choudhri TF, Watters WC III, Resnick DK, Sharan A, et al: Guideline update for the performance of fusion procedures for degenerative disease of the lumbar spine. Part 14: brace therapy as an adjunct to or substitute for lumbar fusion. J Neurosurg Spine 21:91-101, 2014

8. Deyo RA, Mirza SK, Martin BI, Kreuter W, Goodman DC, Jarvik JG: Trends, major medical complications, and charges associated with surgery for lumbar spinal stenosis in older adults. JAMA 303:1259-1265, 2010

9. Dorward IG, Lenke LG, Bridwell KH, O'Leary PT, Stoker GE, Pahys JM, et al: Transforaminal versus anterior lumbar interbody fusion in long deformity constructs: a matched cohort analysis. Spine (Phila Pa 1976) 38:E755-E762, 2013

10. Glassman SD, Copay AG, Berven SH, Polly DW, Subach BR, Carreon LY: Defining substantial clinical benefit following lumbar spine arthrodesis. J Bone Joint Surg Am 90:18391847,2008

11. Glassman SD, Schwab F, Bridwell KH, Shaffrey C, Horton W, Hu S: Do 1-year outcomes predict 2-year outcomes for adult deformity surgery? Spine J 9:317-322, 2009

12. Godil SS, Parker SL, Zuckerman SL, Mendenhall SK, Glassman SD, McGirt MJ: Accurately measuring the quality and effectiveness of lumbar surgery in registry efforts: determining the most valid and responsive instruments. Spine $\mathbf{J}$ 14:2885-2891, 2014

13. Guo HR, Tanaka S, Cameron LL, Seligman PJ, Behrens VJ, Ger J, et al: Back pain among workers in the United States: national estimates and workers at high risk. Am J Ind Med 28:591-602, 1995

14. Guzman JZ, Iatridis JC, Skovrlj B, Cutler HS, Hecht AC, Qureshi SA, et al: Outcomes and complications of diabetes mellitus on patients undergoing degenerative lumbar spine surgery. Spine (Phila Pa 1976) 39:1596-1604, 2014

15. Henry SM, Van Dillen LR, Ouellette-Morton RH, Hitt JR, Lomond KV, DeSarno MJ, et al: Outcomes are not different for patient-matched versus nonmatched treatment in subjects with chronic recurrent low back pain: a randomized clinical trial. Spine J 14:2799-2810, 2014
16. Martin BI, Franklin GM, Deyo RA, Wickizer TM, Lurie JD, Mirza SK: How do coverage policies influence practice patterns, safety, and cost of initial lumbar fusion surgery? A population-based comparison of workers' compensation systems. Spine J 14:1237-1246, 2014

17. Matamalas A, Ramírez M, Mojal S, García De Frutos A, Molina A, Saló G, et al: The visual analog scale and a fiveitem verbal rating scale are not interchangeable for back pain assessment in lumbar spine disorders. Spine (Phila Pa 1976) 35:E1115-E1119, 2010

18. Rajaee SS, Kanim LE, Bae HW: National trends in revision spinal fusion in the USA: patient characteristics and complications. Bone Joint J 96-B:807-816, 2014

19. Solberg T, Johnsen LG, Nygaard OP, Grotle M: Can we define success criteria for lumbar disc surgery?: estimates for a substantial amount of improvement in core outcome measures. Acta Orthop 84:196-201, 2013

20. Stratford PW, Binkley J, Solomon P, Finch E, Gill C, Moreland J: Defining the minimum level of detectable change for the Roland-Morris questionnaire. Phys Ther 76:359-368, 1996

21. Ware JE Jr: SF-36 health survey update. Spine (Phila Pa 1976) 25:3130-3139, 2000

\section{Disclosures}

The authors report no conflict of interest concerning the materials or methods used in this study or the findings specified in this paper.

\section{Author Contributions}

Conception and design: Adogwa. Acquisition of data: Adogwa. Analysis and interpretation of data: Adogwa. Drafting the article: Adogwa, Elsamadicy. Critically revising the article: all authors. Reviewed submitted version of manuscript: all authors. Approved the final version of the manuscript on behalf of all authors: Adogwa. Statistical analysis: Adogwa. Study supervision: Adogwa, Bagley.

\section{Correspondence}

Owoicho Adogwa, Division of Neurosurgery, Duke University Medical Center, Duke South, Blue Zone Rm. 4528, Durham, NC 27710. email: owoicho.adogwa@gmail.com. 